\title{
Cerebrovascular disease associated with Parkinson's disease in Moldovan cohort study
}

\author{
Lilia Rotaru \\ Laboratory of Functional Neurology, Diomid Gherman Institute of Neurology and Neurosurgery \\ Chisinau, the Republic of Moldova \\ Author's ORCID iD, academic degrees and contribution are available at the end of the article \\ Corresponding author - Lilia Rotaru, e-mail: liliarotaru@yahoo.com \\ Manuscript received August 30, 2021; revised manuscript October 29, 2021; published online December 17, 2021
}

\begin{abstract}
Background: Parkinson's disease (PD) is frequently associated with brain vascular lesions (BVLs), which may influence the severity of the disease. Material and methods: BVLs on MRI were determined in 78.4\% of 111 consecutive PD patients (mean age $64.87 \pm 7.69$ y.o.; disease duration $50.21 \pm$ 38.61 mo.; 48 women (43.2\%), 63 men (56.8\%)).

Results: White matter lesions were present in 73 patients (p.) (65.77\%): 61p. (54.95\%) - deep white matter, 46p. (41.44\%) - periventricular white matter, and 41 p. (36.94\%) - both locations. Lacunas were determined in 19p. (17.12\%), cerebral fissures deepening - 52p. (46.85) \%), perivascular spaces dilation - 34p. (30.63\%), ventricular system dilation - 29p. (26.13\%). Patients with and without BVLs had similar ages, ages at PD onset and disease duration. They had insignificantly higher Beck (7.26 \pm 5.62 vs $6.86 \pm 4.34$ ), PDQ39 (Parkinson's Disease Questionnaire) (59.71 \pm 20.38 vs $51.94 \pm 27.69)$ and NMS (Non-Motor Symptoms) $(75.06 \pm 45.21$ vs $71.67 \pm 26.35)$ scores; and lower MoCA (Montreal Cognitive Assessment) scores $(21.92 \pm 4.25$ vs $22.38 \pm$ 4.57). QRISK3 scores ( $19.68 \pm 16.16$ vs $12.90 \pm 6.58)$ and levodopa equivalent daily dose (639.98 \pm 223.05 vs. $439.69 \pm 404.87)$ were significantly higher in patients with BVLs.

Conclusions: Brain vascular lesions were common in our PD patients, and were associated with higher QRISK3 scores and higher levodopa equivalent daily dose, suggesting more disease severity.

Key words: Parkinson's disease, brain vascular lesions
\end{abstract}

\section{Cite this article}

Rotaru L. Cerebrovascular disease associated with Parkinson's disease in Moldovan cohort study. Mold Med J. 2021;64(6):33-36. https://doi.org/10.52418/ moldovan-med-j.64-6.21.06.

\section{Introduction}

Neurodegenerative disorders, such as Parkinson's disease (PD), more frequently occur in the context of other chronic conditions associated with aging. PD and cerebrovascular disease (CVD), both are common in aged populations. PD, as well as brain vascular changes in strategic regions, may display: tremor, rigidity, slowness, gait disturbance, postural instability, urinary incontinence, mood disorders, and cognitive impairment [1]. Cognitive impairment and dementia are recognized features of $\mathrm{PD}$, but these symptoms may relate to comorbid cerebrovascular disease too [2]. Parkinson's disease (PD) is frequently associated with white matter hyperintensities and other brain vascular lesions (BVLs). Studies suggest that the latter may influence the severity of the disease. White matter hyperintensities may be a contributing factor for cognitive impairment [3], as well as to increased motor severity and gait impairment [4].

There are conflicting literature data on PD and CVD association: (1) no clear relationship between PD and CVD [5]; (2) protective effect of PD from CVD [6]; (3) increased risk of CVD in PD [7].

Earlier studies provided no clear relationship between $\mathrm{PD}$ and CVD [5].
Protective effect of PD from CVD may be due to the decreased amount of smoking among PD patients. PD patients seem to experience more of their CVD as TIA than stroke: they have increased access to neurological care, so TIAs are more readily recognized and treated and are therefore less likely to lead to stroke [8]. Dopamine deficiency in PD could ameliorate ischemic damage. It has been found that dopamine depletion with either lesions of the substantia nigra lessen ischemic damage [9].

Increased risk of CVD in PD may be due to the shared pathogeneses between the two diseases or to PD-related effects. Patients with PD should be more aware of the risk of CVD despite having fewer traditional vascular risk factors. In a meta-analysis of four clinical case-control studies, $\mathrm{PD}$ was more associated with CVD (OR: 2.89, 95\% CI: $1.36-$ 6.13); and in three postmortem cohort studies PD patients were at higher risk of CVD during the follow-up period (HR: 1.84, 95\% CI: 1.34-2.54)[7].

\section{Material and methods}

These are preliminary data of a cohort study of Moldovan patients with incident Parkinson's disease. Diagnosis of PD was based on widely acknowledged criteria [10]. Structured 
interview on complaints, medical history and family history of cardiovascular, neurological and psychiatric diseases and drug history was applied; general neurological and medical examinations were conducted. Severity of parkinsonism and disability were assessed by the Modified Unified Parkinson's Disease Rating Scale (MDS-UPDRS) [11]. Cognitive impairment was quantified by Montreal Cognitive Assessement (MoCA) score [12], depression by Beck score [13], non-motor symptoms - by Non-Motor Symptoms score [14], quality of life - by PDQ39 (Parkinson's Disease Questionnaire) score [15].

All brain MRI (magnetic resonance imaging) examinations were performed using a 3.0 T MRI scanner.

MRI Analysis of cerebrovascular disease markers included: lacunas, white matter lesions / hyperintensities, perivascular spaces dilation / enlarged perivascular spices, cerebral fissures deepening, ventricular system dilation.

Lacunas were defined as round or ovoid cerebrospinal fluid-filled cavities in the basal ganglia or white matter, usually 3-15 mm, with low signal on T1WI and DWI, and high signal on T2WI [16]. Periventricular white matter lesions and deep white matter lesions were graded using the Fazekas scale [17], perivascular spaces dilation were defined as punctate hyperintensities on T2WI in the basal ganglia, usually $<3 \mathrm{~mm}$ in diameter [18].

Patients were sub-classified at baseline into two groups according to presence of any of mentioned above cerebrovascular disease as: (1) PD patients with brain vascular lesions; and (2) PD patients without brain vascular lesions.

The data analysis was performed via statistical program Stat-Direct, using descriptive, variation, and correlational analysis. Student's t tests or Mann-Whitney tests were used as appropriate. $P$ values less than 0.05 were considered statistically significant.

BVLs on MRI were determined in $78.4 \%$ of 111 consecutive PD patients, (mean age $64.87 \pm 7.69$ y.o.; disease duration $50.21 \pm 38.61$ mo.; 48 women (43.2\%), 63 men (56.8\%)).

\section{Results and discussion}

The study included 111 consecutive PD patients. The mean age in the cohort was $64.87 \pm 7.69$ years old and disease duration of $50.21 \pm 38.61$ months. In 88 (78.4\%) of our PD patients was determined the presence of brain vascular lesions. White matter lesions were present in 73 patients (65.77\%): 61 of them having (54.95\%) deep white matter lesions, $46(41.44 \%)$ - periventricular white matter lesions, and 41 patients (36.94\%) - a combination of periventricular and deep white matter lesions. Lacunas were determined in 19 patients (17.12\%), cerebral fissures deepening - in 52 patients $(46.85 \%)$, perivascular spaces dilation - in 34 patients $(30.63 \%)$ and ventricular system dilation - in 29 patients (26.13\%).

In Ma X. et al. [19] study, lacunas were found in $9.3 \%$ of patients with $\mathrm{PD}$, periventricular white matter hyperintensities - in $89.7 \%$, deep white matter hyperintensities - in $81.3 \%$, enlarged perivascular spaces - in $85 \%$, and cerebral microbleeds - in 2.8\%. In their study PD patients, showed higher periventricular white matter hyperintensities and deep white matter hyperintensities scores compared with normal controls. Advanced PD patients, versus early PD group exhibited greater periventricular white matter hyperintensities $(\mathrm{P}=0.041)$, deep white matter hyperintensities $(\mathrm{P}=0.046)$, and total cerebral small vessel disease score $(\mathrm{P}$ $=0.044$ ) than the early PD group. In Ma X. et al. [19] study, higher Hoehn\&Yahr stage was independently correlated with increased total cerebral small vessel disease score (OR $=2.667,95 \%$ CI 1.154-2.266) and periventricular white matter hyperintensities score $(\mathrm{OR}=2.237,95 \%$ CI 1.084 1.696).

In this study, patients with and without brain vascular lesions had similar ages $(65.43 \pm 7.64$ vs $61.01 \pm 7.64)$, similar ages at PD onset $(60.95 \pm 8.09$ vs $56.01 \pm 8.59)$ and similar disease duration $(49.98 \pm 36.76$ vs $60.01 \pm 52.31)$.

Examined PD patients with brain vascular lesions had insignificantly higher Beck score (7.26 \pm 5.62 vs $6.86 \pm 4.34)$. PDQ39 (Parkinson's Disease Questionnaire) score (59.71 \pm 20.38 vs $51.94 \pm 27.69)$ and NMS (Non-Motor Symptoms) score $(75.06 \pm 45.21$ vs $71.67 \pm 26.35)$ were slightly higher in patients with brain vascular lesions. MoCA (Montreal Cognitive Assessment) scores (21.92 \pm 4.25 vs $22.38 \pm 4.57)$ were lower in patients with brain vascular lesions compared to the control.

Forbes E. et al. [20], noted the influence of depression and other non-motor signs on cognitive performance of PD patients; they showed that depression scores $(\beta=-0.034$, $\mathrm{P}<0.001)$, along with: Higher Body Mass Index $(\mathrm{BMI})$ $(\beta=-0.009, P=0.039)$, anxiety scores $(\beta=-0.005, P<0.001)$, Epworth Sleepiness scores $(\beta=-0.017, \mathrm{P}=0.003)$, and REM Sleep Behavior Disorder Screening scores $(\beta=-0.037$, $\mathrm{P}<0.001)$ were associated with faster rates of MoCA decline [20]. Shibata et al. suggested a relationship between cognitive decline and increased cerebral small vessel disease score [19]. Increasing age and reduced MoCA scores were associated with increased small vessels disease burden. Logistic regression analyses of their study demonstrated that periventricular white matter hyperintensities, enlarged perivascular spaces in the basal ganglia, and atrophy were predictors of cognitive impairment in PD.

Liu H. et al. [21] meta-analysis had the aim to review systematically and to identify the relationship between the severity and location of white matter hyperintensities (WMHs) and the degree of cognitive decline in patients with PD. PD demented patients had a significantly higher burden of white matter hyperintensities (SMD $=0.8,95 \%$ CI: 0.44 to $1.71, \mathrm{p}<0.0001$ ), especially deep white matter hyperintensities $(\mathrm{SMD}=0.54,95 \% \mathrm{CI}$ : 0.36 to 0.73 , $\mathrm{p}<0.00001)$ and periventricular hyperintensities $(\mathrm{SMD}=$ 0.70, 95\% CI: 0.36 to 1.04, p < 0.0001), than PD-normal cognition patients, regardless of the adjustment of age. Liu $\mathrm{H}$. et al. [21] concluded that WMHs might be imaging markers for cognitive impairment in PD dementia but not in PDmild cognitive impairment, regardless of age, vascular risk factors, or race. 
In the present study QRISK3 scores $(19.68 \pm 16.16$ vs $12.90 \pm 6.58)$ were significantly higher in patients with brain vascular lesions.

High vascular risk is related to impaired scores for: cognition, bradykinesia, axial, postural-instability-gait-disorder and freezing-of-gait score. Furthermore, high vascular risk was identified as a potential predictor of both mild cognitive impairment and dementia in PD. It was found that the presence of more than 2 vascular risk factors was associated with worse UPDRS 3 motor scores (beta coefficient $4.05,95 \%$ confidence interval $1.48,6.61, \mathrm{p}=.002$ ) and with cognitive impairment (ordinal odds ratio 2.24, 95\% confidence interval $1.34,3.74, \mathrm{p}=.002$ ). Presence of white matter leukoaraiosis (but not lacunas) was associated with impaired cognition $(\mathrm{p}=.006)$ and postural instability gait difficulty $(\mathrm{p}=.010)$, [22].

Similarly, in this study, levodopa equivalent daily doses $(639.98 \pm 223.05$ vs $439.69 \pm 404.87)$ were significantly higher in patients with brain vascular lesions - as an indicator for more severe disease in this group.

Cerebrovascular disease may play a critical role in patients with PD. The total cerebral small vessel disease score is a potential neuroimaging marker for monitoring the progression of $\mathrm{PD}$, as the Hoehn\&Yahr stage is independently correlated with the total cerebral small vessel disease score [19]. In Vesely B. et al. [3] review of PD patients with mild cognitive impairment and dementia they had significantly more white matter lesions than the group without mild cognitive impairment and dementia. There was significant relationship between increasing total white matter lesions volume and worse performance on executive function, memory and language. Patients with vascular parkinsonism and dopaminergic denervation have more severe frontal lobe dysfunctions than patients with PD. According to Stojkovic T. et al. [22] results, motor scores were significantly higher in cognitively impaired patients, and only axial score discriminant between mild cognitive impairment and dementia. Whole brain white matter volume was associated with PD dementia, freezing of gait and attention deficits. Additionally, age and bradykinesia scores were independently associated with PD-mild cognitive impairment and age, axial score and whole brain white matter lesions volume with PD-dementia.

\section{Conclusions}

In in the present study cerebrovascular disease was common in MRI evaluated Parkinson's disease patients. Brain vascular lesions were more prevalent in patients with a higher QRISK3 score. PD patients with cerebrovascular comorbidity had higher levodopa equivalent daily dose, suggesting more PD severity.

\section{References}

1. Morley JF, Duda JE. Parkinson's disease and the risk of cerebrovascular pathology. Mov Disord. 2012;27:1471-1472.

2. Shibata K, Sugiura M, Nishimura Y, et al. The effect of small vessel disease on motor and cognitive function in Parkinson's disease. Clin Neurol Neurosurg. 2019;182:58-62. doi: 10.1016/j.clineuro.2019.04.029.
3. Veselý B, Rektor I. The contribution of white matter lesions (WML) to Parkinson's disease cognitive impairment symptoms: a critical review of the literature. Parkinsonism Relat Disord. 2016;22:Suppl 1:S166-S170. doi: 10.1016/j.parkreldis.2015.09.019.

4. Veselý B, Antonini A, Rektor I. The contribution of white matter lesions to Parkinson's disease motor and gait symptoms: a critical review of the literature. J Neural Transm. 2016;123(3):241-250. doi: 10.1007/ s00702-015-1470-9.

5. Jellinger KA. Prevalence of cerebrovascular lesions in Parkinson's disease. A postmortem study. Acta Neuropathol. 2003;105(5):415-419. doi: 10.1007/s00401-003-0676-3.

6. Nanhoe-Mahabier W, de Laat KF, Visser JE, et al. Parkinson's disease and comorbid cerebrovascular disease. Nat Rev Neurol. 2009;5(10):533541. doi: 10.1038/nrneurol.2009.136.

7. Hong CT, Hu HH, Chan L, et al. Prevalent cerebrovascular and cardiovascular disease in people with Parkinson's disease: a meta-analysis. Clin Epidemiol. 2018;10:1147-1154. doi: 10.2147/CLEP.S163493.

8. Nataraj A, Rajput AH. Parkinson's disease, stroke, and related epidemiology. Mov Disord. 2005;20(11):1476-1480. doi: 10.1002/mds.20608.

9. Korten A, Lodder J, Vreeling F, et al. Stroke and idiopathic Parkinson's disease: does a shortage of dopamine offer protection against stroke? Mov Disord. 2001;16(1):119-123. doi: 10.1002/1531-8257(200101)16:1<119::aid-mds1024>3.0.co;2-w.

10. Postuma RB, Poewe W, Litvan I, et al. Validation of the MDS clinical diagnostic criteria for Parkinson's disease. Mov Disord. 2018;33(10):16011608. doi: $10.1002 / \mathrm{mds} .27362$.

11. Goetz CG, Tilley BC, Shaftman SR, et al. Movement Disorder SocietySponsored Revision of the Unified Parkinson's Disease Rating Scale (MDS-UPDRS): Scale presentation and clinimetric testing results. Mov Disord. 2008;23(15):2129-2170. doi: 10.1002/mds.22340.

12. Kasten M, Bruggemann N, Schmidt A, et al. Validity of the MoCA and mmse in the detection of MCI and dementia in Parkinson's disease. Neurology. 2010;75(5):478-479. doi: 10.1212/WNL.0b013e3181e7948a.

13. Visser M, Leentjens AFG, Marinus J, et al. Reliability and validity of the Beck Depression Inventory in patients with Parkinson's disease. Mov Disord. 2006;21(5):668-672. doi: 10.1002/mds.20792.

14. van Wamelen DJ, Martinez-Martin P, Weintraub D, et al. The NonMotor Symptoms Scale in Parkinson's disease: Validation and use. Acta Neurol Scand. 2021;143(1):3-12. doi: 10.1111/ane.13336.

15. Hagell P, Nygren C. The 39 item Parkinson's disease questionnaire (PDQ-39) revisited: Implications for evidence-based medicine. J Neurol Neurosurg Psychiatry. 2007;78(11):1191-1198. doi: 10.1136/ jnnp.2006.111161.

16. Wardlaw JM, Smith EE, Biessels GJ, et al. Neuroimaging standards for research into small vessel disease and its contribution to aging and neurodegeneration. Lancet Neurol. 2013;12(8):822-838. doi: 10.1016/ S1474-4422(13)70124-8.

17. Fazekas F, Chawluk JB, Alavi A. MR signal abnormalities at 1.5 $\mathrm{T}$ in Alzheimer's dementia and normal aging. Am J Neuroradiol. 1987;149(2):351-356. doi: 10.2214/ajr.149.2.351.

18. Doubal FN, MacLullich AMJ, Ferguson KJ, et al. Enlarged perivascular spaces on MRI are a feature of cerebral small vessel disease. Stroke. 2010;41(3):450-454. doi: 10.1161/STROKEAHA.109.564914.

19. Ma X, Li S, Li C, et al. Total cerebral small vessel score association with Hoehn and Yahr stage in Parkinson's disease. Front Aging Neurosci. 2021;13:1-7. doi: 10.3389/fnagi.2021.682776.

20. Forbes E, Tropea TF, Mantri S, et al. Modifiable comorbidities associated with cognitive decline in Parkinson's disease. Mov Disord Clin Pract. 2021;8(2):254-263. doi: 10.1002/mdc3.13143.

21. Liu H, Deng B, Xie F, et al. The influence of white matter hyperintensity on cognitive impairment in Parkinson's disease. Ann Clin Transl Neurol. 2021;8(9):1917-1934. doi: 10.1002/acn3.51429.

22. Stojkovic T, Stefanova E, Soldatovic I, et al. Exploring the relationship between motor impairment, vascular burden and cognition in Parkinson's disease. J Neurol. 2018;265(6):1320-1327. doi: 10.1007/ s00415-018-8838-3. 


\section{Author's ORCID iD and academic degrees}

Lilia Rotaru, MD, PhD, Associate Professor of Neurology - https://orcid.org/0000-0002-5340-5234.

\section{Author's contribution}

LR conceptualized the idea, conducted literature review, wrote the manuscript, revised and finalized the text.

\section{Funding}

The trial was the author's initiative. The author is independent and takes responsibility for the integrity of the data and accuracy of the data analysis.

\section{Ethics approval and consent to participate}

The research project was approved by the Research Ethics Committee of Diomid Gherman Institute of Neurology and Neurosurgery (protocol No 1 of 27.02.2020).

\section{Conflict of Interests}

No competing interests were disclosed.

\section{Note}

The publication of this article is done in the framework of the project: the State Program 2020-2023 “Major cognitive disorders (dementia) in patients with the neurodegenerative and cerebrovascular pathology", 20.800009.8007.39. 